\title{
Geostatistical inference of hydraulic conductivity and dispersivities from hydraulic heads and tracer data
}

\begin{abstract}
Wolfgang Nowak ${ }^{1}$ and Olaf A. Cirpka ${ }^{2}$
Received 22 December 2005; revised 10 April 2006; accepted 25 April 2006; published 10 August 2006.

[1] In groundwater, hydraulic heads and solute arrival times depend primarily on the hydraulic conductivity field and hydraulic boundary conditions. The spread of breakthrough curves, in contrast, depends also on longitudinal and transverse dispersion coefficients. The shape of point-like measured breakthrough curves can be reproduced by simulations only when appropriate dispersivities are applied. The values and spatial distributions of dispersivities depend on the resolution of the underlying hydraulic conductivity field. We present a geostatistical method for the joint estimation of log conductivity and log dispersivities from measurements of hydraulic heads and temporal moments of local breakthrough curves. The parameter fields are considered random space variables, and they are conditioned on the measurements by Bayesian inference. The estimated longitudinal and transverse dispersivities are to be applied in conjunction with the estimated conductivity field. We apply our technique to data of a technical-scale tracer experiment. In the particular application, the amount and quality of measured data are sufficient to infer the conductivity distribution at a spatial resolution at which the spread of locally obtained breakthrough curves is dominated by pore-scale transverse dispersion.
\end{abstract}

Citation: Nowak, W., and O. A. Cirpka (2006), Geostatistical inference of hydraulic conductivity and dispersivities from hydraulic heads and tracer data, Water Resour. Res., 42, W08416, doi:10.1029/2005WR004832.

\section{Introduction}

[2] In the past two decades, several basic approaches to analyze groundwater flow and solute transport in heterogeneous formations have evolved. Many of them are based on stochastic analysis [see Rubin, 2003]. Here, the hydraulic conductivity is considered a random space variable. Since the actual distribution at a site is typically unknown or at least uncertain, exact predictions of flow and transport are impossible. Given the statistics of the parameter field, however, the statistics of hydraulic heads, velocities, and concentrations can be derived. In the stochastic framework, we distinguish between methods that explicitly account for measurements and those that rely on statistical information alone.

[3] In Monte Carlo simulations, typically, a set of random $\log$ conductivity fields is generated. Then, flow and transport is numerically simulated for each field, and the ensemble of different outcomes is analyzed, e.g., by evaluating the mean and variance [e.g., Bellin et al., 1992]. If actual measurements are neglected, Monte Carlo simulations may be seen as the conceptually simplest approach to obtain ensemble averages. However, the computational effort is rather high because second-order statistics of the dependent quantities require thousands of realizations.

[4] In analytical upscaling approaches, heterogeneous media are replaced by equivalent homogeneous media with

\footnotetext{
${ }^{1}$ Institut für Wasserbau, Universität Stuttgart, Stuttgart, Germany.

${ }^{2}$ Swiss Federal Institute of Aquatic Science and Technology, Dübendorf, Switzerland.

Copyright 2006 by the American Geophysical Union. 0043-1397/06/2005WR004832
}

process-specific effective parameters obtained by ensemble averaging. For solute transport in particular, dispersion coefficients were defined to parameterize the spreading and/or dilution of solutes in heterogeneous media. Gelhar and Axness [1983], Neuman et al. [1987], and Dagan [1988] developed expressions for macrodispersivity that apply to large (ergodic) plumes. For small (nonergodic) plumes, Dentz et al. [2000a, 2000b] derived effective dispersion coefficients. Effective dispersion coefficients for point-like injections characterize the dilution of solutes, which is an important property when modeling reactive transport [Cirpka, 2002]. The disadvantage of these approaches is that no actual field data but only some of their geostatistical parameters are included in the upscaling procedure.

[5] In geostatistical inverse modeling, the distribution of hydraulic parameters, such as hydraulic conductivity, is conditioned on measurements of conductivity and dependent quantities. One may distinguish between methods that compute a best estimate and methods to generate conditional realizations. The latter are discussed further below. When computing the best estimate, the most likely value of hydraulic conductivity is determined at each location, together with the conditional covariance that parameterizes the remaining, correlated uncertainty, following a Bayesian framework. Among these techniques are kriging and cokriging [see Hoeksema and Kitanidis, 1984], the successive linear estimator [Yeh et al., 1996], the quasi-linear geostatistical approach [Kitanidis, 1995] and the maximum a posteriori method [McLaughlin and Townley, 1996]. For an extensive review of geostatistical inverse methods, see Zimmerman et al. [1998]. 
[6] Most of these geostatistical inverse methods focus on flow parameters such as hydraulic conductivity. While some of them also consider transport parameters such as sorption coefficients [e.g., Huang et al., 2004]), none of them considers scale-dependent transport parameters, such as dispersivities, to the best of our knowledge. The conditioned conductivity fields cover the variability of aquifers only to a certain extent that depends on the quality and quantity of available data. Hence transport simulations using these fields together with local-scale dispersivities lead to an underestimation of dispersion. However, macrodispersivities also are not applicable because they assume that no variability is resolved, leading to an overestimation of dispersion.

[7] A rigorous discussion of the dispersion of solute clouds in conditional fields leads to nonlocal, computationally demanding formulations due to the inherent nonstationarity of the velocity field [Neuman, 1993]. Neglecting pore-scale dispersion, Rubin [1991] suggested to integrate the conditional velocity covariance along the conditional mean trajectory in order to quantify the dispersion of solutes in conditioned conductivity fields, while Graham and McLaughlin [1989] presented moment-generating equations for concentration statistics in an Eulerian framework.

[8] Some approaches have been derived in which the nonstationarity of partially resolved conductivity fields is neglected in the derivation of dispersivities. Rubin et al. [1999] derived block-effective dispersion coefficients using high-pass filters on the overall velocity covariance, which subsequently is substituted into Dagan's [1988] expression of macrodispersion. Later, Rubin et al. [2003] extended this approach to smaller (nonergodic) plumes. This approach requires that all heterogeneity on the scale of the blocks is resolved and that the remaining uncertainty is much smaller than the block scale. Cirpka and Nowak [2003] adopted the concept of a high-pass filter with a filter function derived from averaged conditional covariance functions for a regular grid of measurement points. Unfortunately, the underlying assumptions of both methods are rather restrictive, so that these approaches are difficult to apply in practice.

[9] Rather than parameterizing the effects of unresolved heterogeneity, one might simulate it by generating conditional realizations using, e.g., the pilot point method by RamaRao et al. [1995], the method of sequential selfcalibration by Gómez-Hernández et al. [1997], or the spectral method by Dietrich and Newsam [1996]. Like for Monte Carlo simulations using unconditional fields, the disadvantage of conditional realizations is that only large ensembles of realizations can adequately represent the unresolved variability. Many realizations are required, and all of them must be conditioned individually, thus resulting in high computational costs.

[10] In the present study, we consider the case that both hydraulic head measurements and breakthrough curves of concentration are available in the conditioning procedure. Then, the information on solute dispersion is included in the concentration data and can be obtained by inverse modeling rather than upscaling. In the next section, we explain our approach and the methods used to implement it. Technical details on a similar method are also given by Nowak [2005]. Finally, we apply our approach to tracer data obtained in a technical-scale sandbox experiment with heterogeneous filling and compare our results to the outcome of other methods of tracer test analysis.

\section{Approach}

[11] We consider the hydraulic conductivity $K$ and the longitudinal and transverse dispersivities $\alpha_{\ell}$ and $\alpha_{t}$ as unknown random space functions, which we identify using an extension of the quasi-linear geostatistical approach of Kitanidis [1995]. We assume porosity to be uniform. In our application, the measurements include values of hydraulic head and temporal moments of breakthrough curves. In general, other types of data such as direct measurements of conductivity or velocities from borehole dilution tests may also be used.

[12] In order to sufficiently resolve the point-like observation scale for breakthrough curves and the spatial structure of the random space variables, we have to choose a rather fine computational resolution of the domain, yielding a large number of discrete values of the unknowns. Additionally, the amount of measurement data is quite high in spite of reducing breakthrough curves to temporal moments. This requires computationally efficient inverse methods.

[13] Local breakthrough curves record how solute clouds pass by an observation point, hence mainly providing information on longitudinal dispersion. The observed degree of longitudinal dispersion consists of two pore-scale contributions: (1) the direct effect of longitudinal pore-scale dispersion and (2) the indirect effect of transverse pore-scale dispersion that transforms longitudinal spreading to longitudinal dilution, a phenomenon which we refer to as aliasing [Cirpka and Kitanidis, 2000b]. Depending on the degree of longitudinal spreading, the first or the second contribution dominates.

[14] Because of this aliasing, single breakthrough curves contain no separable information on transverse and longitudinal local dispersivities. Direct information on transverse dispersion of the solute cloud can only be obtained from very dense transverse curtains of observations, which is impractical and too expensive in practice. Hence we chose to define the dispersivities as $\alpha_{\ell}=\alpha$ and $\alpha_{t}=\lambda \alpha$ and use the magnitude $\alpha$ and the anisotropy ratio $\lambda$ as unknown parameters rather than using $\alpha_{\ell}$ and $\alpha_{t}$. Although this choice may seem to be insignificant at first sight, it installs a defined correlation between $\alpha_{\ell}$ and $\alpha_{t}$ through the anisotropy ratio $\lambda$, alleviating the ill posedness caused by the aliasing effect, regardless of the transverse data spacing. Later on, we will assume that $\alpha$ is an autocorrelated random space function, whereas $\lambda$ will be defined by a linear trend function along the principal flow direction with uncertain drift coefficients.

\subsection{Properties of the Identified Dispersivities}

[15] In the following, $\alpha_{\ell}=\alpha$ and $\alpha_{t}=\alpha \lambda$ denote the dispersivities to be identified. They have the following properties.

[16] 1. They are effective dispersivities for a point-like observation scale in the sense of Dentz et al. [2000a] because we use local, i.e., nearly point-like, measurements of breakthrough curves. Larger support volumes would change the character of $\alpha_{\ell}$ and $\alpha_{t}$ toward larger scales.

[17] 2. They depend on the resolution of the jointly inferred conductivity distribution because they parameterize the 
effect of unresolved heterogeneity. When $\ln K$ fluctuations are neglected entirely, they approach the values of effective dispersivities [Dentz et al., 2000a], whereas they become pore-scale dispersivities, when the $\ln K$ field is fully resolved.

[18] 3. They are specific for the particular boundary conditions chosen for flow and transport in the experiment, e.g., because dispersivities are traveltime dependent.

[19] 4. In accordance with the previous three points, they are not purely a property of the porous medium.

[20] 5. They are spatially fluctuating variables, not only due to the traveltime dependence of dispersivities in general, but also because the degree of resolved heterogeneity varies inside the domain, depending on the sampling strategy, i.e., the distance, quantity, quality and type of adjacent measurements [cf. Rubin, 1991].

[21] 6. We define lognormal prior distributions using the substitutes $\Xi_{\alpha}=\ln \alpha_{\ell}$ and $\Xi_{\lambda}=\ln \lambda$ in the inverse procedure. This assumption is a legitimate and convenient way of ensuring nonnegativity and it is in accordance with the commonly used lognormal distribution of conductivity $K$ with $Y=\ln K$.

[22] 7. For spatial inference, we define $\ln \alpha$ to be multiGaussian, which constitutes a maximum entropy assumption on the type of joint distribution.

[23] 8. The structure, variance and integral scale of the corresponding geostatistical model cannot be determined from experimental variogram analysis of direct measurements but must be inferred from measurements of dependent quantities.

[24] 9. Like in most other geostatistical and interpolation methods, simulated values of measured quantities using the estimated parameters are fitted at the locations of observation and are best estimates in between.

[25] The multivariate log Gaussian distribution and the dependence on model scale and boundary conditions are in accordance with wide-spread assumptions and wellunderstood properties of hydraulic conductivity. The estimated values of dispersivities, however, depend significantly stronger on the resolution of the measurements and on hydraulic boundary conditions than estimates of hydraulic conductivity. This is so, because dispersivities quantify the unresolved velocity fluctuations sampled by a plume along the trajectory from the injection to the observation point. Numerical values about dispersivities always require statements about the associated resolution of velocity. Coarsening the measurement grid would lead to a smoother estimate of the velocity field and consequently to larger estimates of dispersivities. For a more detailed discussion, we refer to Nowak [2005], who identified a scalar log dispersion coefficient that has the same fundamental properties.

\subsection{Identifying Structural Parameters of the Geostatistical Model}

[26] Fortunately, under certain circumstances, the structure of estimated parameter fields is insensitive to the structure and integral scale of the geostatistical model. The prerequisites are, that (1) the data spacing is equal to or smaller than the integral scale and/or (2) the scale of sensitivity patterns is larger than the integral scale [e.g., Kitanidis, 1997]. Both is the case in the application we feature in this study. In the absence of variogram information and direct observations, we optimize the structural parameters based on indirect measurements following the quasi-linear geostatistical approach [Kitanidis, 1995], with an extension to uncertain prior knowledge.

\subsection{Use of Temporal Moments}

[27] We use temporal moments $m_{k}$ instead of entire breakthrough curves $c(t)$ for two reasons. First, the generating equations for temporal moments, equations (9)-(11) are formally identical to steady state equations, avoiding time-consuming computation of the solute concentration history $c(t)$. Second, unlike the concentrations $c(t)$ themselves, the temporal moments $m_{k}$ depend almost monotonically on the parameters of interest. Thus conditioning log conductivity and log dispersivities on temporal moments is a better posed problem than conditioning the same parameters on concentrations [e.g., Ezzedine and Rubin, 1996].

[28] In previous studies [e.g., Harvey and Gorelick, 1995a; Cirpka and Kitanidis, 2000a; James et al., 2000], only the first temporal moments of breakthrough curves, or mean arrival times, were considered for inverse modeling. These are sensitive to the conductivity distribution, but quite insensitive to dispersivities. In the present study, we additionally use second central temporal moments, or spread of breakthrough curves, in order to identify the dispersivities. Hydraulic heads and the first temporal moment of breakthrough curves are the most significant information for identifying hydraulic conductivity as the second central moment is for dispersivities. Still, we condition conductivity also on the second central moment and dispersivities also on the first in order to use as much information from the moments as possible.

[29] For technical reasons that involve the condition of several matrices in our inverse procedure, we scale the second central temporal moment by the first.

\section{Governing Equations}

[30] According to Darcy's law, the specific discharge $\mathbf{q}\left[L T^{-1}\right]$ in a porous medium is:

$$
\mathbf{q}=-K \nabla \phi
$$

in which $K\left[L T^{-1}\right]$ is the hydraulic conductivity, here assumed isotropic on the local scale, and $\phi[L]$ is the hydraulic head. The seepage velocity $\mathbf{v}\left[L T^{-1}\right]$ is related to the specific discharge $\mathbf{q}$ by the flow-effective porosity $\theta[-]$, which here is assumed uniform:

$$
\mathbf{v}=\frac{\mathbf{q}}{\theta}
$$

Under steady state conditions without internal sources and sinks, mass conservation yields the steady state groundwater flow equation:

$$
\nabla \cdot(K \nabla \phi)=0 \quad \text { in } \Omega
$$

with $\Omega$ denoting the domain. We consider the following set of boundary conditions:

$$
\begin{aligned}
(K \nabla \phi) \cdot \mathbf{n}=\hat{q} & \text { on } \Gamma_{1} \\
\phi=\hat{\phi} & \text { on } \Gamma_{2}
\end{aligned}
$$


where $\hat{\phi}$ and $\hat{q}$ are prescribed heads and normal flux components defined on the boundary $\Gamma=\Gamma_{1} \cup \Gamma_{2}$, and $\mathbf{n}$ is the normal unit vector pointing outward.

[31] Transport of a conservative, nonsorbing solute in porous media is described by the advection-dispersion equation:

$$
\frac{\partial c}{\partial t}+\nabla \cdot(\mathbf{v} c-\mathbf{D} \nabla c)=0 \quad \text { in } \Omega
$$

with $c\left[M L^{-3}\right]$ denoting concentration, $t[T]$ being time and D $\left[L^{2} T^{-1}\right]$ the dispersion tensor. Equation (5) is subject to a general set of boundary conditions:

$$
\begin{array}{rlrl}
(\mathbf{v} c-\mathbf{D} \nabla c) \cdot \mathbf{n} & =\hat{c} \mathbf{v} \cdot \mathbf{n} \quad & \text { on } \Gamma_{\mathrm{in}_{1}} \\
c & =\hat{c} & & \text { on } \Gamma_{\mathrm{in}_{2}} \\
(\mathbf{D} \nabla c) \cdot \mathbf{n} & =0 & & \text { on } \Gamma \backslash \Gamma_{\mathrm{in}} .
\end{array}
$$

Here, $\hat{c}$ is a specified concentration, and $\Gamma_{\mathrm{in}_{1}}$ and $\Gamma_{\mathrm{in}_{2}}$ are the inflow sections of the boundary. We restrict the choice of boundary conditions such that $\Gamma_{2}$ and $\Gamma_{\text {in }}$ do not intersect. According to Scheidegger [1961], the dispersion tensor D is:

$$
D_{i j}=\frac{v_{i} v_{j}}{\|\mathbf{v}\|}((1-\lambda) \alpha)+\delta_{i j}\left(\lambda \alpha\|\mathbf{v}\|+D_{m}\right)
$$

where $v_{i}$ is the $i$ th component of the velocity vector, $\alpha_{\ell}=$ $\alpha$ and $\alpha_{t}=\lambda \alpha[L]$ are the longitudinal and transverse dispersivities and $D_{m}\left[L^{2} T^{-1}\right]$ is the pore diffusion coefficient. The Kronecker symbol $\delta_{i j}$ is unity for $i=j$ and zero otherwise.

\subsection{Temporal Moments of Breakthrough Curves}

[32] The $k$ th raw temporal moment $m_{k}\left[M T^{k} L^{-3}\right]$ of a local breakthrough curve $c(\mathbf{x}, t)$ is defined by

$$
m_{k}(\mathbf{x})=\int_{0}^{\infty} t^{k} c(\mathbf{x}, t) d t
$$

According to Steiner's theorem, the second central temporal moment is $m_{2 c}=m_{2}-m_{1}^{2} / m_{0}\left[M T^{2} L^{-3}\right]$. We define a normalized second central moment $m_{2 c n}[T]$ as $m_{2 c n}=$ $m_{2 c} / m_{1}$.

[33] For an extensive overview on the physical meaning of temporal moments for solute transport in heterogeneous formations, we refer to Cirpka and Kitanidis [2000a]. Harvey and Gorelick [1995b] derived moment-generating equations for raw temporal moments of solute concentration. These are partial differential equations that are formally identical to steady state transport equations:

$$
\nabla \cdot\left(\mathbf{v} m_{k}-\mathbf{D} \nabla m_{k}\right)=k m_{k-1} \quad \text { in } \Omega \text {. }
$$

with the boundary conditions:

$$
\begin{aligned}
\left(\mathbf{v} m_{k}-\mathbf{D} \nabla m_{k}\right) \cdot \mathbf{n} & =\mathbf{v} \cdot \mathbf{n} \hat{m}_{k} \quad \text { on } \Gamma_{\mathrm{in}_{1}} \\
m_{k} & =\hat{m}_{k} \quad \text { on } \Gamma_{\mathrm{in}_{2}} \\
\left(\mathbf{D} \nabla m_{k}\right) \cdot \mathbf{n} & =0 \quad \text { on } \Gamma \backslash \Gamma_{\mathrm{in}},
\end{aligned}
$$

in which $\hat{m}_{k}$ is the $k$ th raw temporal moment of $\hat{c}$. For the case of a uniform solute injection, a moment-generating equation of the second central moment $m_{2 c}$ can be defined. It merely has a different source term than that of the corresponding raw moment $m_{2}$ [Cirpka and Kitanidis, 2000b]:

$$
\begin{aligned}
\nabla \cdot\left(\mathbf{v} m_{2 c}\right) & -\nabla \cdot\left(\mathbf{D} \nabla m_{2 c}\right) \\
= & 2\left(\nabla m_{1}\right)^{T} \frac{\mathbf{D}}{m_{0}}\left(\nabla m_{1}\right) .
\end{aligned}
$$

In the advection-dominated case, when the diffusive flux across $\Gamma_{\mathrm{in}_{1}}$ and $\Gamma_{\mathrm{in}_{2}}$ can be neglected, the boundary conditions for equation (11) are identical to those for equation (10).

\section{Geostatistical Inversion}

[34] Under realistic conditions, the parameters $K, \alpha_{\ell}$ and $\alpha_{t}$ in the above equations are not known. We identify the unknown distributions of $\log$ conductivity $Y=\ln K$, longitudinal $\log$ dispersivity $\Xi_{\alpha}=\ln \alpha$ and $\log$ anisotropy ratio $\Xi_{\lambda}=\ln \lambda$ following the quasi-linear geostatistical approach of Kitanidis [1995]. The version we use includes the generalized concept of uncertain mean [Nowak and Cirpka, 2004]. In this study, we extend the approach to include the generalized cases of uncertain prior knowledge about structural parameters. In the following, we give a brief outline of the extended method.

[35] Consider a random $n \times 1$ multi-Gaussian vector of unknowns s (e.g., discrete values of $\ln K, \ln \alpha$ and $\ln \lambda$ ) with expected value $E[\mathbf{s}]=\mathbf{X} \boldsymbol{\beta}$. $\mathbf{X}$ is a $n \times p$ matrix of known deterministic base functions, and $\beta$ is a $p \times 1$ vector of uncertain drift coefficients (e.g., representing a constant mean plus a spatial trend). In the generalized case of uncertain prior knowledge, the drift coefficients are distributed $\boldsymbol{\beta} \sim \mathbf{N}\left(\boldsymbol{\beta}^{*}, \mathbf{Q}_{\boldsymbol{\beta} \beta}\right)$, where $\boldsymbol{\beta}^{*}$ is the uncertain prior value, and $\mathbf{Q}_{\boldsymbol{\beta} \beta}$ quantifies the prior uncertainty of $\boldsymbol{\beta}$ (e.g., the variance of the mean and other trend coefficients). Then, $\mathbf{s}$ is distributed $\mathbf{s} \sim \mathbf{N}\left(\mathbf{X} \boldsymbol{\beta}^{*}, \mathbf{G}_{\mathbf{s s}}\right)$, where $\mathbf{G}_{\mathbf{s s}}=\mathbf{Q}_{\mathbf{s s}}+\mathbf{X} \mathbf{Q}_{\boldsymbol{\beta} \boldsymbol{\beta}} \mathbf{X}^{T}$ is the $n \times n$ generalized autocovariance matrix of $\mathbf{s}$ [cf. Kitanidis, 1993], and $\mathbf{Q}_{\mathbf{s s}}$ is the covariance matrix of $\mathbf{s}$ given $\beta$.

[36] Now, consider the $m \times 1$ vector of error-prone observations $\mathbf{y}$ (here, hydraulic heads and moments of breakthrough curves) related to $\mathbf{s}$ via a nonlinear transfer function $\mathbf{f}$ (here, the flow and transport equations) and an error vector $\mathbf{r}: \mathbf{y}=\mathbf{f}(\mathbf{s})+\mathbf{r}$, with $\mathbf{r} \sim \mathbf{N}(\mathbf{0}, \mathbf{R})$. $\mathbf{R}$ is a $m \times m$ matrix, typically assumed diagonal. In the quasi-linear geostatistical approach, the transfer function is successively linearized about the current estimate $\mathbf{s}_{k}$, and a modified vector of observations is used:

$$
\mathbf{y}_{k}^{\prime}=\mathbf{y}-\mathbf{f}\left(\mathbf{s}_{k}\right)+\tilde{\mathbf{H}}_{k} \mathbf{s}_{k}
$$

in which $\tilde{\mathbf{H}}_{k}$ is the $m \times n$ sensitivity matrix linearized about $\mathbf{s}_{k}$ with $\tilde{H}_{i, j}=\partial f_{i} / \partial s_{j}$. Linearized uncertainty propagation yields that $\mathbf{y}_{k}^{\prime} \sim \mathbf{N}\left(\tilde{\mathbf{H}}_{k} \mathbf{X} \boldsymbol{\beta}^{*}, \tilde{\mathbf{G}}_{\mathbf{y y}, k}\right)$, in which $\tilde{\mathbf{G}}_{\mathbf{y y}, k}=$ $\tilde{\mathbf{H}}_{k} \mathbf{G}_{\mathbf{s s}} \tilde{\mathbf{H}}_{k}^{T}+\mathbf{R}$ is the $m \times m$ generalized autocovariance matrix of $\mathbf{y}_{k}^{\prime}$. 
[37] The best estimate $\hat{\mathbf{s}}$ is defined by the minimum of the objective function:

$$
\begin{aligned}
L(\mathbf{s})= & \left(\mathbf{s}-\mathbf{X} \boldsymbol{\beta}^{*}\right)^{T} \mathbf{G}_{\mathbf{s s}}^{-1}\left(\mathbf{s}-\mathbf{X} \boldsymbol{\beta}^{*}\right) \\
& +(\mathbf{y}-\mathbf{f}(\mathbf{s}))^{T} \mathbf{R}^{-1}(\mathbf{y}-\mathbf{f}(\mathbf{s})) .
\end{aligned}
$$

The best estimate $\hat{\mathbf{s}}$ and the conditional covariance $\mathbf{Q}_{\mathbf{s s} \mid \mathbf{y}}$ are obtained from:

$$
\begin{gathered}
\hat{\mathbf{s}}_{k+1}=\mathbf{X} \widehat{\boldsymbol{\beta}}_{k+1}+\mathbf{Q}_{\mathbf{s s}} \tilde{\mathbf{H}}_{k}^{T} \boldsymbol{\xi}_{k+1} \\
\tilde{\mathbf{Q}}_{\mathbf{s s} \mid \mathbf{y}} \geq \mathbf{G}_{\mathbf{s s}}-\mathbf{G}_{\mathbf{s s}} \tilde{\mathbf{H}}_{k}^{T} \tilde{\mathbf{G}}_{\mathbf{y y}, k}^{-1} \tilde{\mathbf{H}}_{k} \mathbf{G}_{\mathbf{s s}}
\end{gathered}
$$

in which the $p \times 1$ vector $\widehat{\boldsymbol{\beta}}$ of conditional drift coefficients and the $m \times 1$ vector $\xi$ of weights are obtained by solving:

$$
\left[\begin{array}{cc}
\tilde{\mathbf{G}}_{\mathbf{y y} . k} & \tilde{\mathbf{H}}_{k} \mathbf{X} \\
\mathbf{X}^{T} \tilde{\mathbf{H}}_{k}^{T} & -\mathbf{Q}_{\beta \beta}^{-1}
\end{array}\right]\left[\begin{array}{c}
\boldsymbol{\xi}_{k+1} \\
\widehat{\boldsymbol{\beta}}_{k+1}
\end{array}\right]=\left[\begin{array}{c}
\mathbf{y}_{k}^{\prime} \\
-\mathbf{Q}_{\beta \beta}^{-1}\left(\boldsymbol{\beta}^{*}-\widehat{\boldsymbol{\beta}}_{k}\right)
\end{array}\right] .
$$

[38] The orthonormal residuals are important quantities for model criticism [Kitanidis, 1991]. The most efficient way to compute them is [Nowak, 2005]:

$$
\hat{\mathbf{r}}_{n}=\mathbf{S R}^{-1}(\mathbf{y}-\mathbf{f}(\hat{\mathbf{s}}))
$$

where $\mathbf{S}: \mathbf{S}^{T} \mathbf{S}=\tilde{\mathbf{G}}_{\mathbf{y y}}=\tilde{\mathbf{H}}_{k} \mathbf{G}_{\mathbf{s s}} \tilde{\mathbf{H}}_{k}^{T}+\mathbf{R}$ is obtained by Cholesky decomposition.

[39] The best estimate $\hat{\mathbf{s}}$ is based on a parameterization of the solution space using the cross covariance $\mathbf{Q}_{\mathbf{s s}} \tilde{\mathbf{H}}_{k}^{T}$. All but the last iteration steps merely optimize this subspace, and the last iteration step actually represents the act of conditioning. Kitanidis [1995] refers to this procedure as "finding the peak of the integrand". The process of optimizing the subspace is discussed by Nowak and Cirpka [2004].

[40] In practice, we stabilize the iteration procedure in equations (12)-(16) by a modified Levenberg-Marquardt

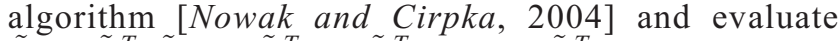
$\tilde{\mathbf{H}}_{k} \mathbf{Q}_{\mathbf{s s}} \tilde{\mathbf{H}}_{k}^{T}, \tilde{\mathbf{H}}_{k} \mathbf{G}_{\mathbf{s s}} \tilde{\mathbf{H}}_{k}^{T}, \mathbf{Q}_{\mathbf{s s}} \tilde{\mathbf{H}}_{k}^{T}$ and $\mathbf{G}_{\mathbf{s s}} \tilde{\mathbf{H}}_{k}^{T}$ using FFT-based methods [Nowak et al., 2003]. For the latter purpose, the unknown parameters must be second-order stationary or at least intrinsic and discretized on a regular grid. The largest matrix to be stored explicitly is sized $m \times n$, and thanks to the function estimate form of the estimator [Kitanidis, 1997], no matrix sized $n \times n$ needs to be inverted. This and further aspects of computational efficiency are discussed by Nowak [2005].

[41] The covariance matrix $\mathbf{Q}_{\mathbf{s s}}$ is usually defined by a geostatistical model, such as the linear, the exponential or the Gaussian ones, with structural parameters $\boldsymbol{\theta}$ (i.e., the integral scales and the variances) that are typically not known a priori. While Kitanidis [1995] identified these parameters from the observations alone, we generalize the approach to the case of uncertain prior knowledge about the structural parameters. As uncertain prior knowledge, we assume the structural parameters $\theta$ to follow a Gaussian distribution with prior mean $\boldsymbol{\theta}^{*}$ and covariance $\mathbf{Q}_{\boldsymbol{\theta} \theta}$. The structural parameters $\theta$ are found by

$$
\begin{aligned}
\boldsymbol{\theta}_{\ell+1}= & \boldsymbol{\theta}_{\ell}-\mathbf{F}^{-1} \mathbf{g} \\
g_{i}= & -\frac{1}{2}\left(\mathbf{y}^{\prime}-\tilde{\mathbf{H}} \mathbf{X} \boldsymbol{\beta}^{*}\right)^{T} \tilde{\mathbf{G}}_{\mathbf{y y}}^{-1} \frac{\partial \tilde{\mathbf{Q}}_{\mathbf{y y}}}{\partial \boldsymbol{\theta}_{i}} \tilde{\mathbf{G}}_{\mathbf{y y}}^{-1}\left(\mathbf{y}^{\prime}-\tilde{\mathbf{H}} \mathbf{X} \boldsymbol{\beta}^{*}\right) \\
& +\frac{1}{2} \operatorname{Tr}\left[\frac{\partial \tilde{\mathbf{Q}}_{\mathbf{y y}}}{\partial \boldsymbol{\theta}_{i}} \tilde{\mathbf{G}}_{\mathbf{y y}}^{-1}\right]+\mathbf{e}_{i}^{T} \mathbf{Q}_{\boldsymbol{\theta}}^{-1}\left(\boldsymbol{\theta}-\boldsymbol{\theta}^{*}\right) \\
F_{i j}= & +\frac{1}{2} \operatorname{Tr}\left[\frac{\partial \tilde{\mathbf{Q}}_{\mathbf{y y}}}{\partial \boldsymbol{\theta}_{i}} \tilde{\mathbf{G}}_{\mathbf{y y}}^{-1} \frac{\partial \tilde{\mathbf{Q}}_{\mathbf{y y}}}{\partial \boldsymbol{\theta}_{j}} \tilde{\mathbf{G}}_{\mathbf{y y}}^{-1}\right]+\mathbf{e}_{i}^{T} \mathbf{Q}_{\boldsymbol{\theta} \boldsymbol{\theta}}^{-1} \mathbf{e}_{j},
\end{aligned}
$$

where $\operatorname{Tr}[\mathbf{A}]$ is the trace of matrix $\mathbf{A}$ and $\mathbf{e}_{i}$ is a unit vector in direction $i$. The original algorithm is obtained for $\mathbf{G}_{\mathbf{y y}}=\mathbf{Q}_{\mathbf{y y}}$ and $\mathbf{Q}_{\theta \theta}^{-1}=0$. For the ease of notation, we omitted the subscript $k$ for the linearized quantities.

\section{Evaluation of Sensitivities}

[42] In the quasi-liner geostatistical approach, we successively linearize the dependence of the observed state variables on the unknown parameters about the current estimate $\hat{\mathbf{s}}_{k}$, applying equation (12). We evaluate the sensitivities using the continuous adjoint state method [Sun and Yeh, 1990].

[43] Consider the current estimates $\tilde{Y}_{k}=\ln \tilde{K}(\mathbf{x}), \tilde{\Xi}_{\alpha}=$ $\ln \tilde{\alpha}(\mathbf{x})$ and $\tilde{\Xi}_{\lambda}=\ln \tilde{\lambda}(\mathbf{x})$, with the dispersion tensor $\tilde{\mathbf{D}}$ obtained from substituting $\tilde{\alpha}$ and $\tilde{\lambda}$ into equation (7). First, we solve the governing equations (3), (9) and (11) for the hydraulic head $\tilde{\phi}(\mathbf{x})$, first moment $\tilde{m}_{1}(\mathbf{x})$, and second central moment $\tilde{m}_{2 c}(\mathbf{x})$ and compute $\tilde{m}_{2 c n}(\mathbf{x})=\tilde{m}_{2 c} / \tilde{m}_{1}$. Subsequently, we solve a set of adjoint state equations for each measurement. The equation for the adjoint state $\psi_{2 c}$ of $m_{2 c}$ is:

$$
\tilde{\mathbf{v}} \cdot \nabla \psi_{2 c}+\nabla \cdot\left(\tilde{\mathbf{D}} \nabla \psi_{2 c}\right)=\delta_{i 2 c} \delta(\mathbf{x}) \text { in } \Omega
$$

subject to the boundary conditions:

$$
\begin{aligned}
\left(\tilde{\mathbf{D}} \nabla \psi_{2 c}\right) \cdot \mathbf{n} & =0 & & \text { on } \Gamma_{\mathrm{in}_{1}} \\
\psi_{2 c} & =0 & & \text { on } \Gamma_{\mathrm{in}_{2}} \\
\left(\tilde{\mathbf{v}} \psi_{2 c}+\tilde{\mathbf{D}} \nabla \psi_{2 c}\right) \cdot \mathbf{n} & =0 & & \text { on } \Gamma \backslash \Gamma_{\mathrm{in}}
\end{aligned}
$$

For the adjoint state $\psi_{1}$ of $m_{1}$ :

$$
\begin{aligned}
\tilde{\mathbf{v}} \cdot & \nabla \psi_{1}+\nabla \cdot\left(\tilde{\mathbf{D}} \nabla \psi_{1}\right) \\
& =4 \nabla \cdot\left(\psi_{2 c} \frac{\tilde{\mathbf{D}}}{m_{0}} \nabla \tilde{m}_{1}\right)+\delta_{i 1} \delta(\mathbf{x}) \text { in } \Omega
\end{aligned}
$$

subject to the boundary conditions:

$$
\begin{aligned}
\left(\tilde{\mathbf{D}} \nabla \psi_{1}\right) \cdot \mathbf{n}=0 & & \text { on } \Gamma_{\mathrm{in}_{1}} \\
\psi_{1}=0 & & \text { on } \Gamma_{\mathrm{in}_{2}} \\
\left(\tilde{\mathbf{v}} \psi_{1}+\tilde{\mathbf{D}} \nabla \psi_{1}\right) \cdot \mathbf{n}=0 & & \text { on } \Gamma \backslash \Gamma_{\text {in }} .
\end{aligned}
$$


For the adjoint state $\psi_{\phi}$ of $\phi$ :

$$
\begin{aligned}
& -\nabla \cdot\left(\tilde{K} \nabla \psi_{\phi}\right)=\nabla \cdot\left(\psi_{2 c} \frac{\tilde{K}}{\theta} \nabla \tilde{m}_{2 c}\right) \\
& +\nabla \cdot\left(\psi_{1} \frac{\tilde{K}}{\theta} \nabla \tilde{m}_{1}\right)+\delta_{i \phi} \delta(\mathbf{x}) \text { in } \Omega
\end{aligned}
$$

subject to the boundary conditions:

$$
\begin{aligned}
\psi_{\phi}= & 0 \text { on } \Gamma_{2} \\
& \left(\psi_{2 c} \frac{\tilde{K}}{\theta} \nabla \tilde{m}_{2 c}+\psi_{1} \frac{\tilde{K}}{\theta} \nabla \tilde{m}_{1}+\tilde{K} \nabla \psi_{\phi}\right) \cdot \mathbf{n}=0 \quad \text { on } \Gamma_{1} .
\end{aligned}
$$

[44] The flag variables $\delta_{i 2 c}, \delta_{i 1}$ and $\delta_{i \phi}$ are unity when measurement $i$ is a measurement of the $m_{2 c}, m_{1}$ or $\phi$, respectively, and $\delta(\mathbf{x})$ is the Dirac delta function. Because of their source terms, these equations have to be solved in the order of $\psi_{2 c}, \psi_{1}$ and $\psi_{\phi}$, starting at the adjoint state of the measurement in question.

[45] Once all relevant adjoint states have been obtained, the sensitivities of the observation $Z$ (either $m_{2 c}, m_{1}$ or $\phi$ ) with respect to piecewise constant values of $\Xi_{\alpha}, \Xi_{\lambda}$ and $Y_{k}$ within the subvolumes $\Omega_{k}$ are given by

$$
\begin{aligned}
\frac{d Z}{d Y_{k}}= & \int_{\Omega_{k}}\left[-\tilde{K} \nabla \tilde{\phi} \cdot \nabla \psi_{\phi}-\psi_{1} \frac{\tilde{K}}{\theta} \nabla \tilde{\phi} \cdot \nabla \tilde{m}_{1}\right. \\
& \left.-\psi_{2 c} \frac{\tilde{K}}{\theta} \nabla \tilde{\phi} \cdot \nabla \tilde{m}_{2 c}\right] d \Omega \\
\frac{d Z}{d \Xi_{k, p}}= & \int_{\Omega_{k}}\left[\nabla \psi_{2 c} \cdot \tilde{\mathbf{D}}_{p} \nabla \tilde{m}_{2 c}-2 \frac{\psi_{2 c}}{m_{0}} \nabla \tilde{m}_{1} \cdot \tilde{\mathbf{D}}_{p} \nabla \tilde{m}_{1}\right. \\
& \left.+\nabla \psi_{1} \cdot \tilde{\mathbf{D}}_{p} \nabla \tilde{m}_{1}\right] d \Omega, \quad p=\alpha, \lambda
\end{aligned}
$$

with

$$
\begin{array}{ll}
\tilde{\mathbf{D}}_{\alpha}: & \tilde{D}_{i j, \alpha}=\frac{v_{i} v_{j}}{\|\mathbf{v}\|}(1-\bar{\lambda}) \bar{\alpha}+\delta_{i j}\|\mathbf{v}\| \bar{\alpha} \bar{\lambda} \\
\tilde{\mathbf{D}}_{\lambda}: & \tilde{D}_{i j, \lambda}=\frac{v_{i} v_{j}}{\|\mathbf{v}\|}(1-\bar{\lambda}) \bar{\alpha}+\delta_{i j}\|\mathbf{v}\| \bar{\alpha} \bar{\lambda}
\end{array}
$$

[46] We discretize all partial differential equations by the Finite Element Method using bilinear elements. For equations (3) and (23), we choose the standard Galerkin method, while we use the streamline upwind Petrov-Galerkin method [Brooks and Hughes, 1982] for equations (9), (11), (19), and (21). As solver, we employ the UMFPACK v4.4 package [Davis, 2004]. The code has been implemented in MATLAB and runs on a dual-processor $2 \times 2.8 \mathrm{GHz}$ desktop computer with 4GB RAM, operating under Linux.

\section{Application to Experimental Data}

[47] We apply our method to evaluate log conductivity and $\log$ dispersivity distributions to data obtained from a large-scale sandbox experiment on longitudinal effective dispersion in heterogeneous porous media conducted by Jose et al. [2004]. Since the experiment is described in detail in the original publication, we summarize it here only briefly.

[48] The sandbox has dimensions of $14 \mathrm{~m} \times 0.13 \mathrm{~m} \times$ $0.5 \mathrm{~m}$ (length $\times$ width $\times$ height). The front face is equipped with glass panels for visual observation of tracer migration. The left and right faces are connected to an inlet and an outlet chamber. The box is filled heterogeneously with four sand mixtures in lenses with average length of $3 \mathrm{~m}$ and height of $0.08 \mathrm{~m}$. The filling procedure resembled a sedimentation process, leading to microstructures within the lenses shown in Figure 1. Table 1 contains hydraulic conductivity values of the sand mixtures which were obtained by permeameter tests. Figure $2 \mathrm{a}$ shows the distribution of hydraulic conductivity using the mapped distribution of sand types and the conductivity values listed in Table 1. This information was not included in the inversion, but is used here as a reference.

[49] As shown in Figures 2e-2g, a dense grid of 100 piezometers (measuring steady state hydraulic heads) and 126 fiber-optic probes (obtaining point-like breakthrough curves of a fluorescent dye tracer) was installed. The boundary conditions for flow are a constant flux of $3 \ell / h$ under confined conditions resulting in a head difference of approximately $3.5 \mathrm{~cm}$ across the box. Initially, the box contained tracer-free water. From time zero on, a fluorescent tracer solution was continuously injected over the entire inflow section until complete breakthrough was observed in the outflow of the domain.

\subsection{Data Processing}

[50] The breakthrough curves of Jose et al. [2004] correspond to continuous injection. In order to compute temporal moments for a pulse-like injection, we analyzed the temporal derivative of the curves after normalization, noise filtered the curves, truncated them as shown in Figure 3 and then computed the moments using equation (8). Mathematical details are provided by Jose et al. [2004]. In addition to the latter study, we removed drift effects and other systematic fluctuations that were common to all probes prior to data processing and applied curve-specific values of the filter strength, resulting in a better quality of the data set.

[51] The uncertainty of the moments was assumed to scale with the signal-to-noise ratio of the individual curves. The average ratio was about 20 , and average values of the relative error are listed in Table 2. Our improved data processing lead to a reliable high-quality data set. The quantity of data is relatively good when considering that there is roughly one measurement of each $\phi, m_{1}$ and $m_{2 c n}$ per correlation length of the conductivity field in $x$ direction and $y$ direction. Only the quality of the pressure data is moderate at best, since they suffer from a poor signal-tonoise ratio. At a measurement error of $\sigma_{\phi}=0.5 \mathrm{~mm}$ evaluated from duplicate statistics and a total pressure difference of $3 \mathrm{~cm}$, the measurement error is merely a factor of 5 smaller than the observed deviations of pressure from its macroscopically expected value.

\subsection{Geostatistical Setup}

[52] For the unknown parameter fields $Y=\ln K$ and $\Xi_{\alpha}=$ $\ln \alpha$, we assume the exponential geostatistical model with uncertain integral scales and variances. In contrast to other 


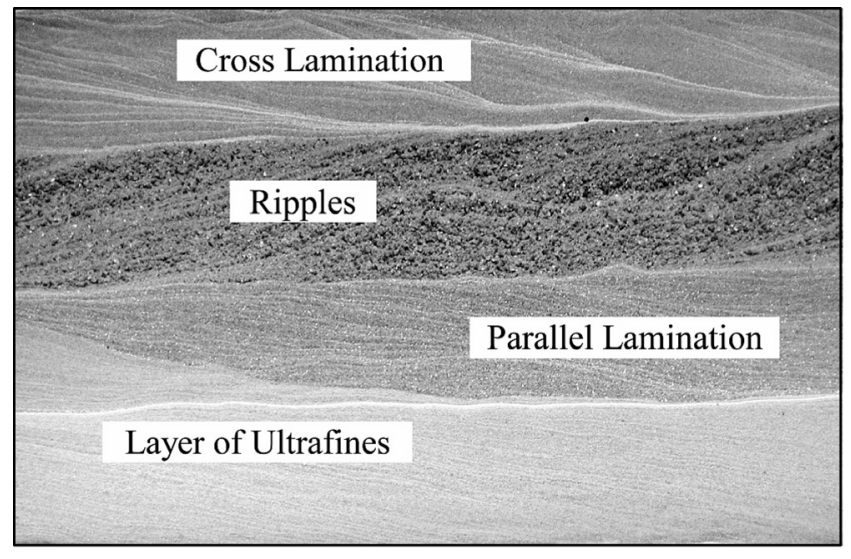

Figure 1. Photograph of microstructures within the sand lenses, exemplary $1 \mathrm{~m}$ long section of the sandbox.

geostatistical models, this choice allows for both finite variance and variability at small scales. The identification of these structural parameters is discussed further below. The structural parameters shown in Table 2 are final values conditioned on the measurements at the end of the inverse procedure. The prior mean values of the fields are uncertain constants $\beta_{Y}^{*}$ and $\beta_{\Xi}^{*}$ with variances $Q_{\beta \beta, Y}$ and $Q_{\beta \beta, \Xi \alpha}$. Additionally, the prior mean of $\ln \alpha$ has an uncertain linear trend along the flow direction since effective longitudinal dispersion coefficients have been shown to increase with travel distance [e.g., Dentz et al., 2000a].

[53] The logarithmic anisotropy ratio $\Xi_{\lambda}=\ln \lambda$ is not treated as an autocorrelated random space variable, i.e., it is fully described by a deterministic trend model with uncertain coefficients. Because transverse dispersivities $\alpha_{t}$ scale differently with traveltime than longitudinal coefficients $\alpha_{\ell}$ [e.g., Dentz et al., 2000a], we allow for an uncertain constant value plus an uncertain linear trend for $\Xi_{\lambda}$. Table 2 displays all parameter values and prior information used for conditioning. The prior mean value of $\ln K$ was estimated from permeameter tests performed with the materials used in the experiment, and the prior mean of $\ln \alpha$ was chosen to range between a characteristic grain size of the materials and the effective dispersivity according to Dentz et al. [2000a]. We assume porosity to be uniform.

[54] We assessed the prior value of the correlation lengths of $\ln K$ from the filling pattern of the sandbox. The prior variance of $\ln K$ and $\ln \alpha$ as well as the correlation lengths of $\ln \alpha$ are estimated from the measured data following equation (18). Prior estimates were $\sigma_{Y}^{2}=2, \sigma_{\alpha}^{2}=2, \lambda_{\alpha x}=$ $2 \mathrm{~m}$ and $\lambda_{\alpha y}=0.025 \mathrm{~m}$ with a relative uncertainty of $50 \%$. The latter three values merely represent an uncertain educated guess: Dilution is mainly driven by zones of intensive shear flow which tend to be longer and thinner than the transverse and longitudinal correlation length scales of $\ln K$, respectively. We assume the structure of $\ln \alpha$ to be in accordance with the geometry of such zones. Indeed, the identified posterior longitudinal correlation length for $\ln \alpha$ will turn out to be larger than that for $\ln K$ while the transverse one is smaller. Identified values that did not differ significantly from the prior estimates were set back to the prior values.
[55] As listed in Table 2, the domain is discretized by $2800 \times 200=560,000$ finite elements with dimensions $5 \mathrm{~mm} \times 2.5 \mathrm{~mm}$ (length $\times$ height $).$

\section{Results}

[56] The estimation of the unknown parameters converged after approximately twenty iteration steps. Thanks to the modified Levenberg-Marquardt algorithm, no oscillations or instabilities occurred. The estimation of structural parameters took three iteration steps and converged to values not far from the prior estimates. Overall, the inverse model ran for about one week. Given the fine resolution of the domain, the computations had been strictly impossible without the acceleration by FFT-based methods [Nowak et al., 2003].

[57] Figures $2 \mathrm{~b}-2 \mathrm{~d}$ and $2 \mathrm{e}-2 \mathrm{~g}$ show the estimated parameter fields and simulated state variables, respectively. Table 3 includes some average values of physical parameters computed from the results of the inversion. Although the unknowns used in the inverse procedure were $\Xi_{\alpha}=\ln \alpha$ and $\Xi_{\lambda}=\ln \lambda$, we show $\alpha_{\ell}=\exp \left(\Xi_{\alpha}\right)$ and $\alpha_{t}=\exp \left(\Xi_{\alpha}\right) \exp \left(\Xi_{\lambda}\right)$ instead, because they are physically more meaningful.

\subsection{Uncertainty of Estimation}

[58] The uncertainty of estimation is quantified by $\mathbf{Q}_{\mathbf{s s} \mid \mathbf{y}}$, see equation (15). The standard deviations of estimation $\sigma_{\text {est }}=\operatorname{sqrt}\left(\operatorname{diag}\left(\mathbf{Q}_{\mathbf{s s} \mid \mathbf{y}}\right)\right)$, which include the conditional uncertainty of the drift coefficients, are shown in Figures $2 \mathrm{~h}-2 \mathrm{j}$. Together with other relevant posterior quantities, Table 3 lists the mean estimation variances normalized by the prior generalized variances, which include the uncertainty of the drift coefficients, $\bar{\sigma}_{\text {est }}^{2}=$ mean $\left(\operatorname{diag}\left(\mathbf{Q}_{\mathbf{s s} \mid \mathbf{y}}\right) / \operatorname{diag}\left(\mathbf{G}_{\mathbf{s s}}\right)\right)$.

[59] Apparently, the overall uncertainty of estimation is much smaller for $\ln K$ than for $\ln \alpha$ and $\ln \lambda$. We attribute this to the high number of measurements for $\phi$ and $m_{1}$ that are highly sensitive to $\ln K$. Especially $\hat{Q}_{\beta \beta Y}$ is almost zero due to the high amount of information on $m_{1}$, whereas information on the magnitude of dispersivity is mainly restricted to measurements of $m_{2 c n}$, resulting in a higher value of $\hat{Q}_{\beta \beta \Xi}$. The anisotropy ratio is quite uncertain because no type of measurement contains direct information on the ratio.

[60] Measurements of $m_{1}$ and $m_{2 c n}$ are most informative at the measurement locations and in a thin up-gradient section along the respective streamline. Measurements of $\phi$, by contrast, have a dipole-shaped sensitivity pattern, being most informative in two distinct zones at a certain

Table 1. Sand Types and Comparison to Estimated $\ln K$ Field: Grain Size and Hydraulic Conductivity Values Determined by Permeameter Tests Compared to Zonal Averages and Standard Deviations Computed From the Estimated Field for Each Material

\begin{tabular}{lcccccc}
\hline & & \multicolumn{2}{c}{$\begin{array}{c}\text { Permeameter } \\
\text { Test }\end{array}$} & & \multicolumn{2}{c}{$\begin{array}{c}\text { Estimated } \\
\text { ln } K\end{array}$} \\
\cline { 3 - 4 } \cline { 7 - 8 } Sand Type & Grain Size, $\mathrm{mm}$ & $K, \mathrm{~m} / \mathrm{s}$ & $\ln K$ & & Mean & $\mathrm{SD}$ \\
\hline Coarse & $1.0-2.5$ & $1.67 \times 10^{-2}$ & -4.09 & & -4.39 & 0.84 \\
Mixed & $0.3-1.2$ & $4.32 \times 10^{-3}$ & -5.44 & & -5.43 & 0.80 \\
Medium & $0.0-3.0$ & $9.09 \times 10^{-4}$ & -7.00 & & -5.39 & 0.98 \\
Fine & $0.1-0.8$ & $5.61 \times 10^{-4}$ & -7.49 & & -5.99 & 0.82 \\
Sandbox & average & $1.75 \times 10^{-3}$ & -6.34 & & -5.42 & 1.03 \\
\hline
\end{tabular}




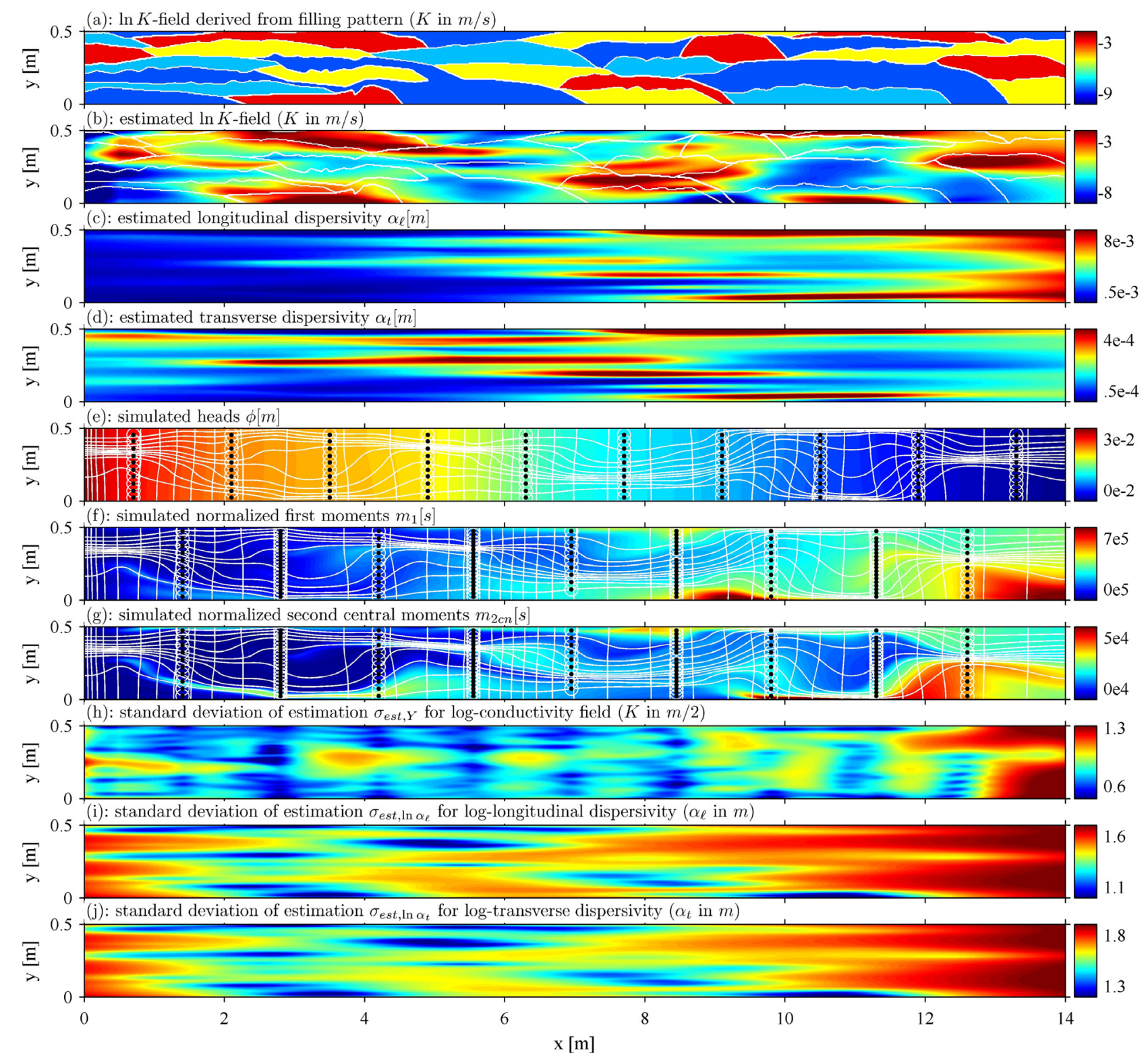

Figure 2. (a) Log conductivity according to filling pattern of the sandbox. (b-d) Estimated Parameter fields $\ln K, \alpha_{\ell}=\exp \left(\Xi_{\alpha}\right)$, and $\alpha_{t}=\exp \left(\Xi_{\lambda}\right) \exp \left(\Xi_{\alpha}\right)$; white lines, flow net; black and white dots, locations of piezometric probes in $\phi$ and fiber-optic probes in $m_{1}$ and $m_{2 c n}$. (e-g) Simulated $\phi, m_{1}$, and $m_{2 c n}$ (h-j) Standard deviation of estimation for $\ln K, \ln \alpha_{\ell}$, and $\ln \alpha_{t}$.

distance up and down gradient of the measurement location. Additionally, the quality of $\phi$ measurements was only moderate, so that the remaining uncertainty in $\ln K$ is very low where breakthrough curves were available, whereas measurements of $\phi$, in our case, hardly have any impact (see Figure $2 \mathrm{~h}$ ). The sensitivity pattern of $m_{1}$ and $m_{2 c n}$ with respect to $\ln \alpha$ is much more complex, so that a direct impact of the sampling network on the respective estimation variance is not apparent in Figures $2 \mathrm{i}$ and $2 \mathrm{j}$.

[61] The different types of parameters were defined to be uncorrelated prior to conditioning. After conditioning, the mean value of $\ln K$ and the drift coefficients for $\ln \alpha$ and $\ln \lambda$ remained uncorrelated, which we attribute to the fact that their main sources of information $\left(m_{1}\right.$ and $m_{2 c n}$ respectively) are independent from each other. By contrast, the conditional mean values and trend coefficients of $\ln \alpha$ and $\ln \lambda$ were anticorrelated with correlation coefficients of $c_{\alpha \lambda \text {,mean }}=-0.87$ and $\mathrm{t} c_{\alpha \lambda \text {,trend }}=-0.37$, respectively. This was to be expected because measurements of $m_{2 c n}$ are positively sensitive to both of them.

\subsection{Statistical Tests}

[62] Both the value of the objective function $L=279.10$ according to equation (13) and the sum of squares of the orthonormal residuals $\left\|\hat{\mathbf{r}}_{n}\right\|_{2}=346.65$ according to equation (17) should follow the $\chi^{2}$ distribution with $m=$ 346 degrees of freedom. Both quantities pass a one-sided statistical test based on a $95 \%$ confidence interval. The fact that $\left\|\hat{\mathbf{r}}_{n}\right\|_{2}$ is almost exactly identical to their expected value of $m=346$ shows that the identified structural parameters fit 

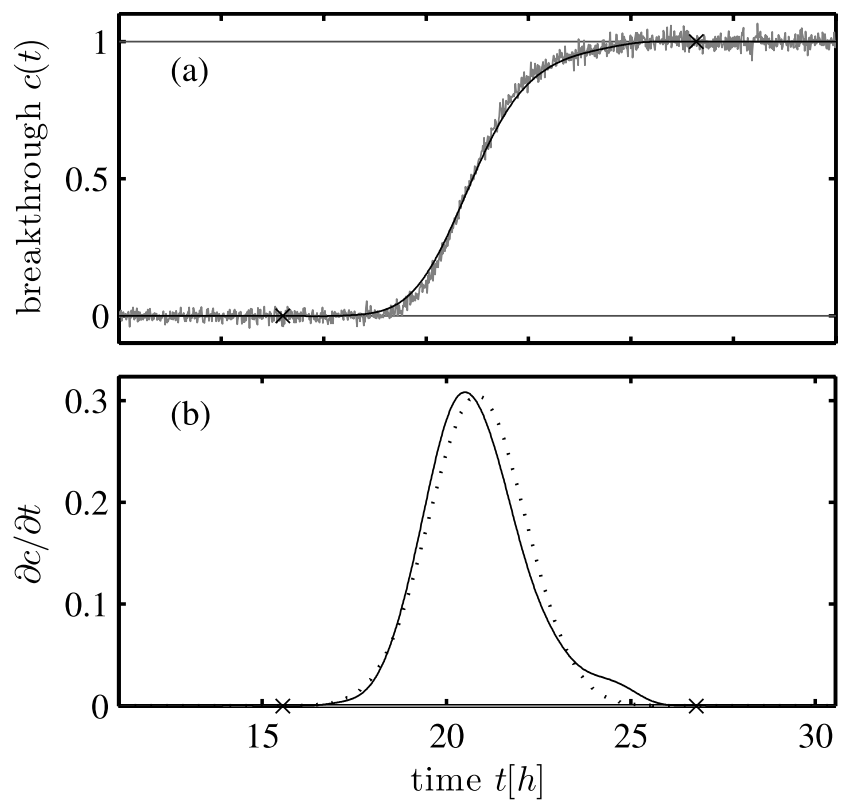

Figure 3. (a) Experimental (gray) and filtered (black) breakthrough curve. (b) Temporal derivative corresponding to instantaneous injection (solid) and equivalent Gaussian curve (dotted) visualizing the first and second central temporal moments. Crosses are truncation points.

well. The orthonormal residuals shown in Figure 4a should behave like white noise, and the plot exhibits only slight evidence of correlation. Comparing their histogram to a normalized Gaussian distribution (see Figure 4b) shows a good visible fit. A corresponding $\chi^{2}$ test for comparison to a Gaussian distribution fails on a $95 \%$ because the tails are not correct, but the inner $90 \%$ of the data pass the test. These results let us have a high trust in the validity of the parameter estimates.

\subsection{Comparison to the Filling Pattern}

[63] Figures $2 \mathrm{a}$ and $2 \mathrm{~b}$ allow a direct comparison between $\ln K$ values obtained by mapping the pattern of the sandbox filling, assigning the values listed Table 1, and those estimated by our inverse method. The white contour lines superimposed in Figure $2 \mathrm{~b}$ represent the different zones shown in Figure 2a. Most areas of high and low estimated conductivity are in accordance with the conductivity field suggested by the filling pattern. For each material type in the filling pattern, we computed the mean and standard deviation of the estimated field in the respective zones and compare them to the permeameter values listed in Table 1. While the zonal averages and the permeameter values correlate with $r^{2}=0.90$, the total sandbox filling as shown in Figure $2 \mathrm{a}$ and the estimated field correlate only with $r^{2}=0.51$. Also, the intrazone standard deviations listed in Table 1 are conspicuously high, almost approaching the standard deviation of the entire estimated field. There are several reasons why the filling pattern is not reproduced more accurately: (1) The conductivity values listed in Table 1 were obtained by permeameter tests which may not be representative for the filling procedure used in the sandbox. Instead, the microstructures and especially the thin layers of ultrafines might be the hydraulically dominating structures overriding the impact of the sand lenses. Additionally, the experimentalists observed clogging within the first meter of the box; (2) the information conveyed by the measurements of head and tracer moments is sparse, imperfect and only indirect, rendering the problem setup ill-posed prior to geostatistical regularization; (3) the filling pattern is a deterministic zonation pattern that does not necessarily succumb to the multi-Gaussian structure assumption and a best estimate, if not including zonation information in the geostatistical setup, can per definition not reproduce sharp outlines. Considering these restrictions, we take the high correlation of zonal values as a strong affirmation of our estimated conductivity field.

\subsection{Measured and Simulated Breakthrough Curves}

[64] Using a transient transport model, we simulated the measured breakthrough curves applying the estimated parameter fields. While the temporal moments used for conditioning, $m_{1}$ and $m_{2 c n}$, agree within a tolerance specified by the geostatistical setup, other features of the breakthrough curves like the skewness and kurtosis cannot be expected to do so. Matching $m_{2 c n}$ is a result of jointly inferring conductivity and dispersivities, where the dispersivities make up for the specific lack of variability in the conductivity field.

Table 2. Parameters and Prior Knowledge Used for the Experimental Data Set

\begin{tabular}{|c|c|c|}
\hline Parameter & Units & Value \\
\hline \multicolumn{3}{|c|}{ Definition of Domain } \\
\hline Domain length $L_{x}$ & $\mathrm{~m}$ & 14 \\
\hline Domain length $L_{y}$ & $\mathrm{~m}$ & 0.5 \\
\hline Grid spacing $d_{x}$ & $\mathrm{~m}$ & $5 \times 10^{-3}$ \\
\hline Grid spacing $d_{y}$ & $\mathrm{~m}$ & $2.5 \times 10^{-3}$ \\
\hline Transport parameters & & \\
\hline Diffusion coefficient $D_{m}$ & $\mathrm{~m}^{2} / \mathrm{s}$ & $10^{-10}$ \\
\hline Porosity $n_{e}$ & & 0.4 \\
\hline \multicolumn{3}{|c|}{ Log Conductivity (Y) } \\
\hline Variance $\sigma_{Y}^{2}$ & & 2 \\
\hline Correlation length $\lambda_{Y, x}$ & $\mathrm{~m}$ & 1 \\
\hline Correlation length $\lambda_{Y, y}$ & $\mathrm{~m}$ & 0.05 \\
\hline Prior mean $\beta_{Y}^{*}$ & & -6 \\
\hline Uncertainty $Q_{\beta \beta Y}$ & & 1 \\
\hline \multicolumn{3}{|c|}{ Log Dispersivity $(\alpha)$} \\
\hline Variance $\sigma_{\Xi \alpha}^{2}$ & & 2 \\
\hline Correlation length $\lambda_{\Xi_{x}}$ & $\mathrm{~m}$ & 2 \\
\hline Correlation length $\lambda_{\Xi y}$ & $\mathrm{~m}$ & 0.025 \\
\hline Prior mean $\beta_{\Xi \alpha}^{*}$ & & -5 \\
\hline Uncertainty $Q_{\beta \beta, \Xi \alpha}$ & & 1 \\
\hline Trend over $14 \mathrm{~m}$ & & $2 / 14$ \\
\hline Uncertainty of trend & & $1 / 14^{2}$ \\
\hline \multicolumn{3}{|c|}{ Log Dispersivity $(\lambda)$} \\
\hline Prior mean $\beta_{\Xi \lambda}^{*}$ & & -2 \\
\hline Uncertainty $Q_{\beta 3, \Xi \lambda}$ & & 1 \\
\hline Trend over $14 \mathrm{~m}$ & & $-2 / 14$ \\
\hline Uncertainty of trend & & $1 / 14^{2}$ \\
\hline \multicolumn{3}{|c|}{ Measurements } \\
\hline Number for $\phi$ & & 100 \\
\hline Average error $\sigma_{\phi}$ & $\mathrm{m}$ & $5 \times 10^{-4}$ \\
\hline Number for $m_{1}$ & & 123 \\
\hline Average error $\sigma_{m 1}$ & $\%$ & 5 \\
\hline Number for $m_{2 c n}$ & & 123 \\
\hline Average error $\sigma_{m 2 c n}$ & $\%$ & 7.5 \\
\hline
\end{tabular}


Table 3. Posterior Parameter Values and Corresponding Mean Properties $^{\mathrm{a}}$

\begin{tabular}{|c|c|}
\hline Parameter & Value \\
\hline \multicolumn{2}{|c|}{$\log$ Conductivity $(Y=\ln K)$} \\
\hline Relative average estimated variation $\bar{\sigma}_{\text {est }, Y}^{2}$ & 0.27 \\
\hline Posterior mean $\hat{\beta}_{Y}$ & -5.67 \\
\hline Posterior uncertainty $\hat{Q}_{\beta \beta Y}$ & 0.065 \\
\hline \multicolumn{2}{|c|}{ Log Dispersivity $\left(\Xi_{\alpha}=\ln \alpha\right)$} \\
\hline Relative average estimated variation $\bar{\sigma}_{e s t, \Xi \alpha}^{2}$ & 0.65 \\
\hline Posterior mean $\hat{\beta}_{\Xi_{\alpha}}$ & -5.84 \\
\hline Posterior uncertainty $\hat{Q}_{33, \Xi \alpha}$ & 0.45 \\
\hline Trend over $14 \mathrm{~m}$ & 2.03 \\
\hline Uncertainty of trend & 0.69 \\
\hline \multicolumn{2}{|c|}{$\log$ Dispersivity $\left(\Xi_{\lambda}=\ln \lambda\right)$} \\
\hline Relative average estimated variation $\bar{\sigma}_{\text {est }, \Xi \lambda}^{2}$ & 0.50 \\
\hline Posterior mean $\hat{\beta}_{\Xi \lambda}$ & -2.66 \\
\hline Posterior uncertainty $Q_{3 \beta, \Xi \lambda}$ & 0.48 \\
\hline Trend over 14 m & -1.75 \\
\hline Uncertainty of trend & 0.73 \\
\hline \multicolumn{2}{|c|}{ Derived Mean Physical Parameters } \\
\hline$K_{g}=\exp \left(\hat{\beta}_{Y}\right)$ & $3.5 \times 10^{-3} \mathrm{~m} / \mathrm{s}$ \\
\hline$v_{g}=Q / A / n_{e}$ & $\begin{array}{c}3.3 \times 10^{-5} \mathrm{~m} / \mathrm{s} \\
2.9 \times 10^{-3} \mathrm{~m}\end{array}$ \\
\hline $\begin{array}{l}\alpha_{\ell, g}=\exp \left(\beta_{\Xi \alpha}\right) \\
D_{\ell, g}=\alpha_{\ell, g} v_{g}\end{array}$ & $\begin{array}{c}2.9 \times 10^{-3} \mathrm{~m} \\
9.7 \times 10^{-8} \mathrm{~m}^{2} / \mathrm{s}\end{array}$ \\
\hline$\alpha_{t, g}=\alpha_{\ell} \exp \left(\hat{\beta}_{\Xi \lambda}\right)$ & $2.0 \times 10^{-4} \mathrm{~m}$ \\
\hline$D_{t, g}=\alpha_{t, g} v_{g}$ & $6.8 \times 10^{-9} \mathrm{~m}^{2} / \mathrm{s}$ \\
\hline
\end{tabular}

${ }^{\mathrm{a}}$ Subscript $g$ indicates geometric means.

[65] In a series of hypothetical test cases (not shown here), we demonstrated that, with a sufficient number of measurement locations and accuracy of the first and second central moments, it is possible to determine the entire system so well that even higher-order moments of the breakthrough curves are matched, although they have not
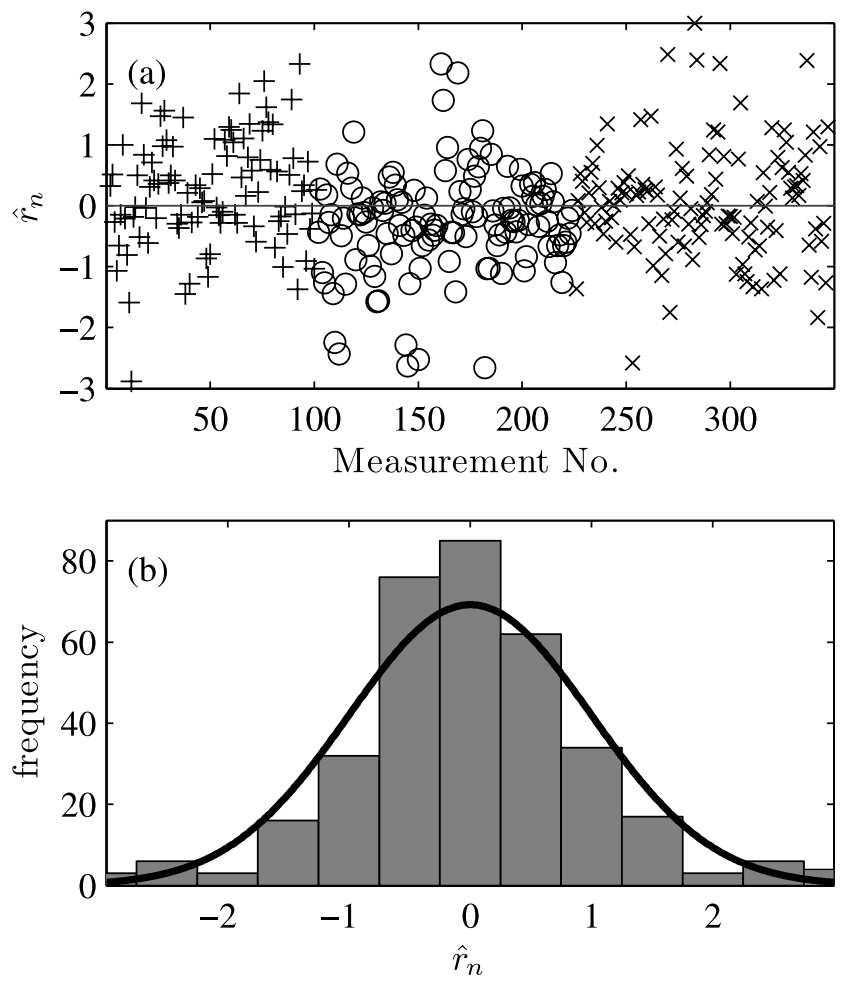

Figure 4. Orthonormal residuals for experimental data set. Pluses, $\phi$; circles, $m_{1}$; crosses, $m_{2 c n}$.
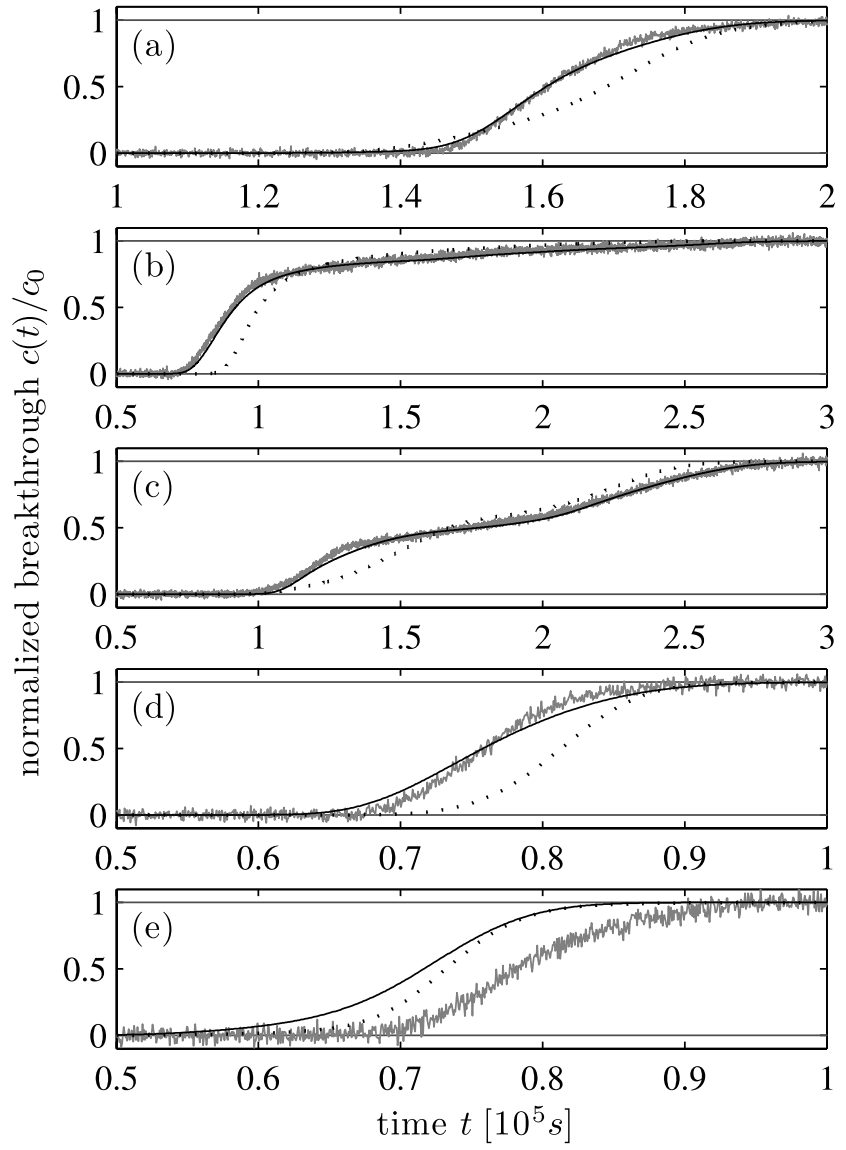

Figure 5. Comparison of normalized measured (gray) and estimated (black) breakthrough curves. Solid lines show the results of our new method that includes measurements of $m_{2 c n}$ and jointly infers spatially variable dispersivities. Dotted lines show the results when not including measurements of $m_{2 c n}$ and using averaged spatially uniform dispersivities.

been used for conditioning [Nowak, 2005]. Figure 5 compares the measurement and simulation for some exemplary breakthrough curves. Figure 5a shows the breakthrough curve at $(x, y)=(4.2 \mathrm{~m}, 0.275 \mathrm{~m})$ where $m_{1}$ and $m_{2 c n}$ is matched well and higher-order features are irrelevant. Figures $5 \mathrm{~b}$ and $5 \mathrm{c}$ show curves at $(x, y)=(4.2 \mathrm{~m}$, $0.075 \mathrm{~m})$ and $(x, y)=(7.0 \mathrm{~m}, 0.175 \mathrm{~m})$ where heavy tailing and multistep breakthrough were observed. Both are reproduced by the simulated curves although tailing and multistep behavior represent skewness (third moment) and kurtosis (fourth moment). Figures $5 \mathrm{~d}$ and $5 \mathrm{e}$ show the curves at $(x, y)=(2.8 \mathrm{~m}, 0.375 \mathrm{~m})$ and $(x, y)=(2.8 \mathrm{~m}, 0.4 \mathrm{~m})$ where arrival time and spreading are off by $50 \%$ and $10 \%$, respectively. As the majority of breakthrough curves were simulated well even in their higher-order moments, we conclude that the data quality and quantity was sufficiently high to obtain reliable parameter estimates and a good physical characterization of the sandbox.

\subsection{Comparison to Other Dispersion Coefficients Obtained for the Sandbox}

[66] Jose et al. [2004] computed the apparent effective dispersion coefficient $D_{\ell}^{a}$ according to Cirpka and Kitanidis 
[2000b] for each measurement location. $D_{\ell}^{a}$ is based on the interpretation of a breakthrough curve by one-dimensional advective-dispersive transport with uniform coefficients. In the sandbox experiment, the cross-sectional average of $D_{\ell}^{a}$ increases from $D_{\ell}^{a}=0.75 \times 10^{-5} \mathrm{~m}^{2} / \mathrm{s}$ at the inlet to $D_{\ell}^{a}=$ $2.75 \times 10^{-5} \mathrm{~m}^{2} / \mathrm{s}$ at the outlet of the sandbox. The same authors also fitted the model of effective dispersion by Dentz et al. [2000a] to the measured data and obtained a local scalar dispersion coefficient of $D_{l o c}=8.24 \times$ $10^{-9} \mathrm{~m}^{2} / \mathrm{s}$ with a factor of variation $F V=2.72$. Theoretically, while $D_{\ell}^{a}$ is a quantity related to a uniform macroscopic flow field with the largest value, our $D_{\ell}$ should be smaller since it applies to the specific degree of resolved variability in the jointly estimated $\ln K$ field, whereas $D_{l o c}$ should be smallest since it represents only pore-scale dispersion, and $D_{t}$ should be in the same order as $D_{l o c}$. In practice, our mean value of $D_{t}=6.8 \times 10^{-9} \mathrm{~m}^{2} / \mathrm{s}$ is in reasonable agreement with $D_{l o c}=8.24 \times 10^{-9} \mathrm{~m}^{2} / \mathrm{s}$. The difference is insignificant when considering the relative uncertainties of both quantities. Also, our mean value of $D_{\ell}=9.7 \times 10^{-8} \mathrm{~m}^{2} / \mathrm{s}$ is three orders smaller than $D_{\ell}^{a}$ and only one order larger than $D_{l o c}$, which substantiates our conclusion that the greatest part of variability has been resolved.

[67] Rahman et al. [2005] conducted an experiment on transverse dispersion in the same setup by injecting a tracer solution only into the lower half of the inflow boundary. These authors computed an apparent transverse dispersivity $\alpha_{t}^{a}$ by fitting an analytical solution of the advection-dispersion equation to highly resolved vertical concentration profiles obtained by digital image processing. They discussed that the distorted streamline pattern displayed in Figure 2 makes it impossible to infer transverse-dispersion coefficients from concentration profiles alone. After correcting for the meandering of streamlines, they obtained effective transverse dispersivities with a mean value of $\alpha_{t}^{a}=$ $0.06 \mathrm{~mm}$ and a coefficient of variation of $69 \%$. This is roughly a third of our mean $\alpha_{t}=0.20 \mathrm{~mm}$. From the length of a reactive plume, Rahman et al. [2005] estimated an effective transverse dispersivity of $0.05 \mathrm{~mm}$. Our estimate is larger. However, in our estimation the factor of uncertainty for $\alpha_{t}$, defined as $\exp \left(\sigma_{e s t, \ln \alpha_{t}}\right)$, is 4.5 . Thus the various values are within the range of estimation uncertainty.

\subsection{Quality of System Characterization}

[68] For the given quality and quantity of the measured data, higher-order features of breakthrough curves are reproduced, although they have not been used as measured data. Also, the system is sufficiently characterized to explain mechanisms behind the hot spots of dilution in the work of Jose et al. [2004], identified by extreme probewise values of $D_{\ell}^{a}$. These are at $(x, y)=(4.2 \mathrm{~m}$, $0.075 \mathrm{~m}),(x, y)=(7 \mathrm{~m}, 0.25 \mathrm{~m}),(x, y)=(9.75 \mathrm{~m}, 0.25 \mathrm{~m})$ and $(x, y)=(12.6 \mathrm{~m}, 0.4 \mathrm{~m})$. The hot spot at $(x, y)=(4.2 \mathrm{~m}$, $0.075 \mathrm{~m})$, for example, is attributed to a highly diluted part of the plume that is detected at the second probe from the bottom because it moved upward following the bend of the streamlines just before the probe (see Figure 2 at $(x, y)=(4.2 \mathrm{~m}$, $0.075 \mathrm{~m})$ ). The dilution itself stems from transverse exchange of fast and slow moving water along the relevant streamline starting at the inlet. The result of transverse exchange is the long tailing of the corresponding breakthrough curve in Figure 5b.
[69] The hot spot at $(x, y)=(7 \mathrm{~m}, 0.25 \mathrm{~m})$ can be explained by an early and a late arriving part of the plume that is mixed in an area of shear flow just before the probe (see Figure 2 at $(x, y)=(7 \mathrm{~m}, 0.25 \mathrm{~m}))$. The mixing of early and late arrival can be seen in the corresponding breakthrough curve in Figure 5c.

[70] For a lower quality and quantity of measurements, this quality of system characterization cannot be expected. With decreasing quality and quantity of data, the underlying flow field will be estimated only poorly which makes it difficult to estimate transport parameters. With a lower spatial resolution of observation points, one could still try to include higher-order moments in the conditioning procedure in order to increase the quantity of information. However, measuring higher-order moments may be highly prone to error. Also, with insufficient information about the underlying flow process, the risk of misinterpretation is high.

\subsection{Advantage of Jointly Estimating Spatially Variable Dispersivities}

[71] Figure 6 shows scatterplots of simulated versus measured first and normalized second central moments. Trends resulting from uniform values of $K, \alpha_{\ell}$ and $\alpha_{t}$ have been removed from the data. In Figure 6, we compare our results to those of the method by Cirpka and Kitanidis [2000a], where the first moments are the only information of the breakthrough curves used in the inversion procedure and only the log conductivity is estimated. In our method, we additionally consider the spread of the breakthrough curves which allows to jointly estimate dispersivities as random spatial variables. For comparison, we applied their method in conjunction with spatially uniform dispersivities computed from our estimate.

[72] The correlation coefficient $r^{2}$ for $m_{1}^{\prime}$ is not affected, since conductivity is the most significant parameter for the arrival time of a solute. The scatterplots of $m_{2 c n}^{\prime}$ show two effects. First, the correlation coefficient for $m_{2 c n}^{\prime}$ improves from $r^{2}=0.27$ for the old method to $r^{2}=$ 0.90 when using our new method with the jointly estimated spatially variable dispersivities, because dispersivities are the most significant parameters for simulating the spread of point-like measured breakthrough curves. $r^{2}$ does not rise higher due to the error assigned to the measurements. Second, the overall magnitude of simulated dilution is too low for the old method by a factor of roughly two. Because the additional information conveyed by the measurements of $m_{2 c n}$ has not been used, less variability is resolved in the conductivity field. This illustrates how the required values of the dispersivities depend on the quantity of measurement data. While the latter effect could be alleviated by fitting values for the uniform dispersivities, the former would not improve. A series of test cases (not discussed here) shows that, with decreasing quality and quantity of measurement data, the significance of conductivity in the simulation of dilution decreases while that of the jointly identified dispersivities increases [Nowak, 2005].

[73] Also, the overall quality of system characterization increases when including measurements of $m_{2 c n}$ and jointly estimating spatially variable dispersivities. In Figure 5, the dotted lines corresponding to the old method fit less accurately. Aside from a poorer overall fit of spread and 

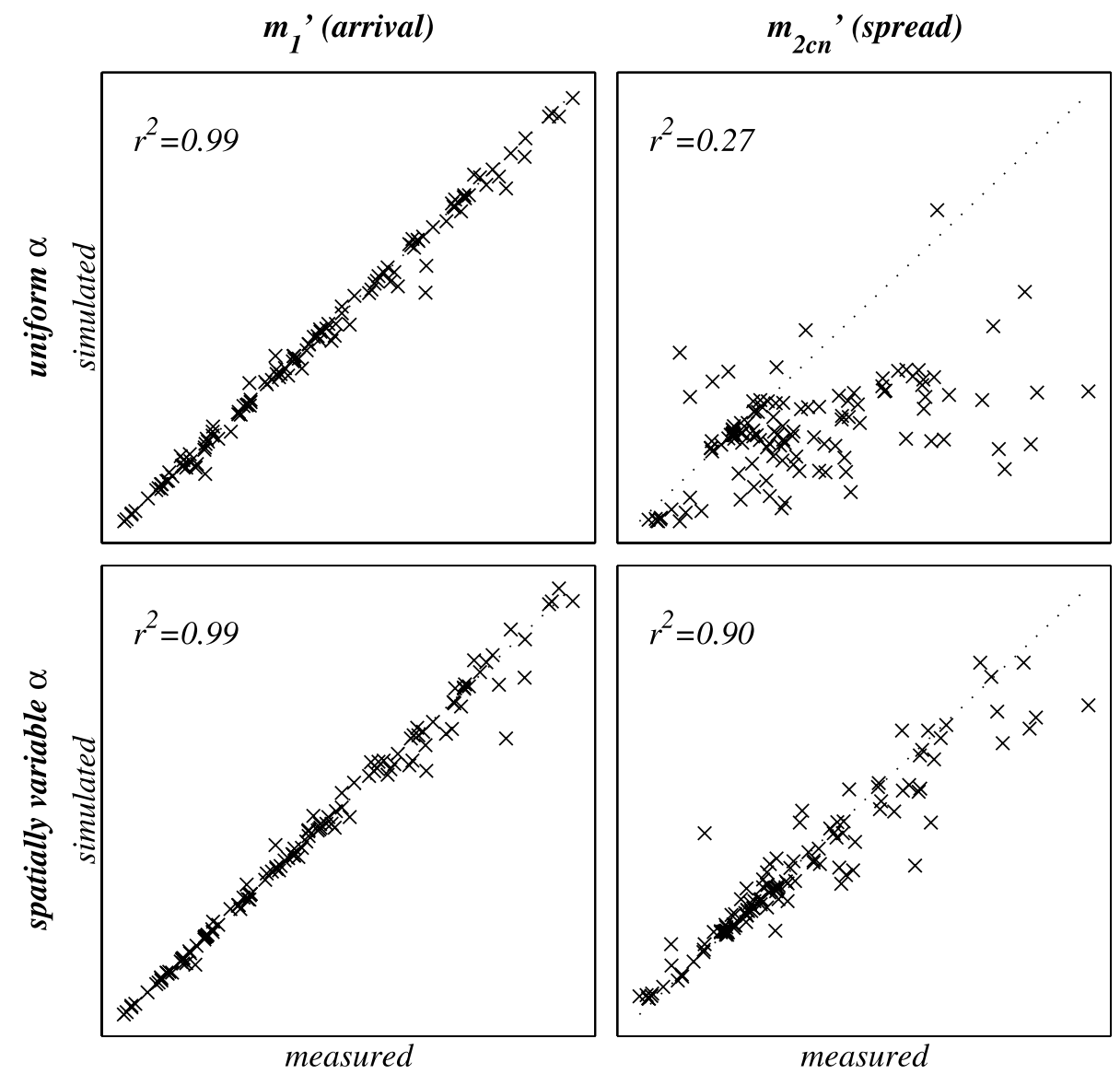

Figure 6. Scatterplots of measured versus simulated values for (left) $m_{1}^{\prime}$ and (right) $m_{2 c n}^{\prime}$. Primes indicate the difference to simulated values using uniform best fit values for $K, \alpha_{\ell}$, and $\alpha_{t}$. (top) "Uniform $\alpha$," illustrating the performance of the predecessor method by Cirpka and Kitanidis [2000a] that does not use measurements of $m_{2 c n}$ and does not estimate spatially variable dispersivities. (bottom) "Spatially variable $\alpha$," illustrating the performance of our new method that additionally uses measurements of $m_{2 c n}$ and jointly infers spatially variable dispersivities.

sometimes even arrival time, the curves show inappropriate fronting, tailing and bimodal breakthrough.

\section{Discussion and Conclusions}

[74] In this study, we successfully developed a method for geostatistical inference of hydraulic conductivity and dispersivities from hydraulic heads and tracer data and applied it to the data set of a tracer experiment performed in a intermediate-scale sandbox [Jose et al., 2004]. The basis of our inverse method is the quasi-linear geostatistical approach [Kitanidis, 1995], accelerated by FFT methods [Nowak et al., 2003], generalized to uncertain prior knowledge about the unknown parameters and stabilized by a modified Levenberg-Marquardt algorithm [Nowak and Cirpka, 2004]. Here, we added a generalization to uncertain prior knowledge about the structural parameters of the geostatistical model. Our method is an extension of the geostatistical inverse method of Cirpka and Kitanidis [2000a], who inferred the log hydraulic conductivity field from measurements of heads and first temporal moments of concentration. In contrast to their method, we also consider measurements of second central temporal moments and jointly estimate log dispersivities.
[75] The identified spatially variable dispersivity fields make up for the specific lack of variability in the corresponding identified conductivity field so that the spread of measured breakthrough curves is reproduced by simulations. In the presented application to experimental data, the estimate withstands statistical tests, the identified conductivity field compares reasonably well to the filling pattern of different sand types used in the experiment, and the estimated transverse dispersivities are in the range of values determined by other methods for the sandbox [Jose et al., 2004; Rahman et al., 2005]. A comparison of measured and simulated breakthrough curves revealed that the system is sufficiently described by the parameter estimate to even reproduce tailing and multistep behavior of single breakthrough curves.

[76] The estimated dispersivities are foremost an interpretation of the data obtained under specific boundary conditions. Like most dispersivities, they are not purely a material property of the formation. They depend on the resolution scales of both the estimated hydraulic conductivity field and the concentration measurements. Conceptionally, they express the unresolved particle velocity fluctuations sampled by the solute plume along its trajectory. Thus reverting the direction of flow would 
require also to revert the trend of the longitudinal dispersivity field. The limited transferability to different hydraulic boundary conditions lies in the nature of solute dispersion. Dispersivities obtained by calibrating deterministic flowand-transport models suffer from the same shortcoming, but are more restricted because they require hard prior knowledge about the structure of the medium.

[77] We see two alternatives to estimating dispersivities together with the best estimate of the conductivity field. The first one would be to restrict the estimation procedure to the conductivities and interpret the spread of breakthrough curves by stochastic theory as is the case, e.g., in the studies of Rubin [1991], Rubin et al. [1999, 2003], and Cirpka and Nowak [2003]. This approach cannot reproduce the observed spread of single breakthrough curves, as it deals only with expected values; it requires crucial assumptions about pore-scale dispersion, e.g., that the pore-scale coefficients are uniform; and it is computationally demanding because of the inherent nonstationarity of conditional log conductivity fields, unless drastic simplifications are made. Thus we doubt that such an approach is manageable and leads to results that can be used in practice.

[78] The second alternative would be to generate conditional realizations of both hydraulic conductivity and porescale dispersivity fields. While one may claim that such an approach leads to parameter fields representing possible physical realities, which depend less on the specific hydraulic boundary conditions than our estimated dispersivity fields, the computational effort is huge: The computational costs for conditioning a single realization are comparable to those of directly finding the best estimate. In the presented two-dimensional application, already 560,000 finite elements were needed to simulate transport accurately enough to estimate dispersivities. In threedimensional applications, millions of elements would be required. Under these conditions, it appears unrealistic to generate thousands of conditional realizations which are needed to obtain reliable second-order conditional statistics.

[79] The data set used in the present study allows for a high spatial resolution of the hydraulic conductivity field. For example, the mechanisms leading to single hot spots of apparent dilution observed by Jose et al. [2004] could be revealed. For the given resolution, pore-scale transverse dispersion was identified as the major process responsible for dilution. If we had used fewer breakthrough curves to infer the conductivity distribution, we had missed important features, the simulated traveltimes had become more uniform perpendicular to the direction of flow, and the observed spread of the remaining breakthrough curves had to be explained by enhanced longitudinal dispersion. This stresses once more the necessity for putting effort into good and large data sets when characterizing flow and transport in heterogeneous systems.

[80] For potential field application, the point-like observation scale seems to be impedimentary since the numerical resolution should be sufficiently fine to resolve the observation scale. The method can be extended to deal with larger support volumes of the breakthrough curves. In this case, however, the identified dispersivities do not represent local-scale dilution but dispersion at the scale of observation. Another approach would be to stochastically describe the subgrid variability in order to decouple the numerical resolution from the observation scale.

[81] The new approach proved to have the ability of handling large data sets and fine spatial resolutions due to a combination of computationally efficient methods. Among these are the reduction of breakthrough curves to temporal moments for efficient handling of timedependent measurements and FFT-based methods for error propagation.

[82] Acknowledgments. This study has been funded by the Deutsche Forschungsgemeinschaft within the Emmy-Noether-program under the grants $\mathrm{Ci}$ 26/3-2 to Ci 26/3-4. We are indebted to Surabhin Chackiath Jose and Arifur Rahman for the experimental data. We thank Anisur Rahman for his contributions to the transport code. We also thank T.-C. J. Yeh and an anonymous reviewer for their constructive suggestions to improve the manuscript.

\section{References}

Bellin, A., P. Salandin, and A. Rinaldo (1992), Simulation of dispersion in heterogeneous porous formations: Statistics, first-order theories, convergence of computations, Water Resour. Res., 28, 2211-2227.

Brooks, A. N., and T. J. R. Hughes (1982), Streamline upwind/PetrovGalerkin formulations for convection dominated flows with particular emphasis on the incompressible Navier-Stokes equations, Comput. Methods Appl. Mech. Eng., 32, 199-259.

Cirpka, O. A. (2002), Choice of dispersion coefficients in reactive transport calculations on smoothed fields, J. Contam. Hydrol., 58, 261-282.

Cirpka, O. A., and P. K. Kitanidis (2000a), Sensitivity of temporal moments calculated by the adjoint-state method, and joint inversing of head and tracer data, Adv. Water Resour, 24, 89-103.

Cirpka, O. A., and P. K. Kitanidis (2000b), Characterization of mixing and dilution in heterogeneous aquifers by means of local temporal moments, Water Resour. Res., 36, 1136-1221.

Cirpka, O. A., and W. Nowak (2003), Dispersion on kriged hydraulic conductivity fields, Water Resour. Res., 39(2), 1027, doi:10.1029/ 2001WR000598.

Dagan, G. (1988), Time-dependent macrodispersion for solute transport in anisotropic heterogeneous aquifers, Water Resour. Res., 24, 1491-1500.

Davis, T. A. (2004), Algorithm 832: UMFPACK v.4.3-An unsymmetricpattern multifrontal method, Trans. Math. Software, 30, 196-199.

Dentz, M., H. Kinzelbach, S. Attinger, and W. Kinzelbach (2000a), Temporal behaviour of a solute cloud in a heterogeneous porous medium: 1. Point-like injection, Water Resour. Res., 36, 3591-3604.

Dentz, M., H. Kinzelbach, S. Attinger, and W. Kinzelbach (2000b), Temporal behaviour of a solute cloud in a heterogeneous porous medium: 2 . Spatially extended injection, Water Resour. Res., 36, 3605-3614.

Dietrich, C. R., and G. N. Newsam (1996), A fast and exact method for multidimensional Gaussian stochastic simulations: Extension to realizations conditioned on direct and indirect measurements, Water Resour. Res., 32, 1643-1652.

Ezzedine, S., and Y. A. Rubin (1996), A geostatistical approach to the conditional estimation of spatially distributed solute concentration and notes on the use of tracer data in the inverse problem, Water Resour. Res., $32,853-862$.

Gelhar, L. W., and C. L. Axness (1983), Three-dimensional stochastic analysis of macrodispersion in aquifers, Water Resour. Res., 19, 161-180.

Gómez-Hernández, J. J., A. Sahuquillo, and J. E. Capilla (1997), Stochastic simulation of transmissivity fields conditional to both transmissivity and piezometric data: 1. Theory, J. Hydrol., 203, 162-174.

Graham, W., and D. McLaughlin (1989), Stochastic analysis of nonstationary subsurface solute transport: 2. Conditional moments, Water Resour. Res., 25, 2331-2355.

Harvey, C. F., and S. M. Gorelick (1995a), Mapping hydraulic conductivity: Sequential conditioning with measurements of solute arrival time, hydraulic-head, and local conductivity, Water Resour. Res., 31, $1615-1626$.

Harvey, C. F., and S. M. Gorelick (1995b), Temporal moment-generating equations: Modeling transport and mass-transfer in heterogeneous aquifers, Water Resour. Res., 31, 1895-1911.

Hoeksema, R. J., and P. K. Kitanidis (1984), An application of the geostatistical approach to the inverse problem in two-dimensional groundwater modeling, Water Resour. Res., 20, 1003-1020. 
Huang, H., X. Hu, X.-H. Wen, and C. Shirley (2004), Stochastic inverse mapping of hydraulic conductivity and sorption partitioning coefficient fields conditioning on nonreactive and reactive tracer test data, Water Resour. Res., 40, W01506, doi:10.1029/2003WR002253.

James, A. I., W. D. Graham, K. Hatfield, P. S. C. Rao, and M. D. Annable (2000), Estimation of spatially variable residual nonaqueous phase liquid saturations in nonuniform flow fields using partitioning tracer data, Water Resour. Res., 36, 999-1012.

Jose, S. C., M. A. Rahman, and O. A. Cirpka (2004), Large-scale sandbox experiment on longitudinal effective dispersion in heterogeneous porous media, Water Resour. Res., 40, W12415, doi:10.1029/2004WR003363.

Kitanidis, P. K. (1991), Orthonormal residuals in geostatistics: Model criticism and parameter estimation, Math. Geol., 23, 741-758.

Kitanidis, P. K. (1993), Generalized covariance functions in estimation, Math. Geol., 25, 525-540.

Kitanidis, P. K. (1995), Quasi-linear geostatistical theory for inversing, Water Resour. Res., 31, 2411-2419.

Kitanidis, P. K. (1997), Introduction to Geostatistics, Cambridge Univ. Press, New York.

McLaughlin, D., and L. R. Townley (1996), A reassessment of the groundwater inverse problem, Water Resour. Res., 32, 1131-1161.

Neuman, S. P. (1993), Eulerian-Lagrangian theory of transport in spacetime nonstationary velocity fields: Exact nonlocal formalism by conditional moments and weak approximation, Water Resour. Res., 29, 633645.

Neuman, S. P., C. L. Winter, and C. M. Newman (1987), Stochastic theory of field-scale Fickian dispersion in anisotropic porous media, Water Resour. Res., 23, 453-466.

Nowak, W. (2005), Geostatistical methods for the identification of flow and transport parameters in subsurface flow, Ph.D. thesis, Inst. für Wasserbau, Univ. Stuttgart, Stuttgart, Germany. (Avaialable at http:/ elib.uni-stuttgart.de/opus/frontdoor.php?source_opus=2275).

Nowak, W., and O. A. Cirpka (2004), A modified Levenberg-Marquardt algorithm for quasi-linear geostatistical inversing, Adv. Water Resour., 27, 737-750.

Nowak, W., S. Tenkleve, and O. A. Cirpka (2003), Efficient computation of linearized cross-covariance and auto-covariance matrices of interdependent quantities, Math. Geol., 35, 53-66.
Rahman, M. A., S. C. Jose, W. Nowak, and O. A. Cirpka (2005), Experiments on vertical transverse mixing in a large-scale heterogeneous model aquifer, J. Contam. Hydrol., 80, 130-148.

RamaRao, B. S., A. M. LaVenue, G. de Marsily, and M. G. Marietta (1995), Pilot point methodology for automated calibration of an ensemble of conditionally simulated transmissivity fields: 1 . Theory and computational experiments, Water Resour. Res., 31, 475-493.

Rubin, Y. (1991), Prediction of tracer plume migration in heterogeneous porous media by the method of conditional probabilities, Water Resour. Res., 27, 1291-1308.

Rubin, Y. (2003), Applied Stochastic Hydrogeology, Oxford Univ. Press, New York.

Rubin, Y., A. Sun, R. Maxwell, and A. Bellin (1999), The concept of block-effective macrodispersivity and a unified approach for grid-scaleand plume-scale-dependent transport, J. Fluid Mech., 395, 161-180.

Rubin, Y., A. Bellin, and A. E. Lawrence (2003), On the use of block-effective macrodispersion for numerical simulations of transport in heterogeneous formations, Water Resour. Res., 39(9), 1242, doi:10.1029/2002WR001727.

Scheidegger, A. E. (1961), General theory of dispersion in porous media, J. Geophys. Res., 66, 3273-3278.

Sun, N.-Z., and W. W.-G. Yeh (1990), Coupled inverse problems in groundwater modeling: 1 . Sensitivity analysis and parameter identification, Water Resour. Res., 26, 2507-2525.

Yeh, T.-C. J., M. Jin, and S. Hanna (1996), An iterative stochastic inverse method: Conditional effective transmissivity and hydraulic head fields, Water Resour. Res., 32, 85-92.

Zimmerman, D. A., et al. (1998), A comparison of seven geostatistically based inverse approaches to estimate transmissivities for modeling advective transport by groundwater flow, Water Resour. Res., 34, $1373-1413$.

O. A. Cirpka, Swiss Federal Institute of Aquatic Science and Technology (Eawag), Überlandstrasse 133, CH-8600 Dübendorf, Switzerland. (olaf. cirpka@eawag.ch)

W. Nowak, Institut für Wasserbau, Universität Stuttgart, Pfaffenwaldring 61, D-70550 Stuttgart, Germany. (wolfgang.nowak@iws.uni-stuttgart.de) 